\title{
Desempenho agronômico de genótipos de amendoim na região da Alta Paulista
}

Submetido - 04 jul. $2020 \quad$ Aprovado - 07 ago. $2020 \quad$ Publicado - 14 out. 2020
dol ${ }_{\text {http://dx.doi.org/10.17648/sas.v1i1.5 }}$

Victor Hugo Lanças Zammataro (iD

Casul Peanuts - Cooperativa Agropecuária de Parapuã, Parapuã, SP, victorhugo@casul.com.br. Jair Heuert (iD
Programa de Melhoramento do Amendoim - Embrapa, Santo Antônio de Goiás, GO,
jair.heuert@embrapa.br.
Lúcio Lima Rodrigues (iD
Discente de Engenharia Básica da UNIP, Goiânia, GO, luciolimarodrigues@gmail.com.
Maurício Lou Danelutti ID Casul Peanuts - Cooperativa Agropecuária de Parapuã, Tupã, SP, mauricio@casul.com.br.
Taís de Moraes Falleiro Suassuna (iD Programa de Melhoramento do Amendoim - Embrapa, Santo Antônio de Goiás, GO, tais.suassuna@embrapa.br.

\begin{abstract}
RESUMO
A cultura do amendoim possui grande relevância na atividade agroindustrial na região da Alta Paulista no estado de São Paulo. O objetivo deste trabalho foi avaliar o desempenho agronômico de dez genótipos de amendoim, na região da Alta Paulista, munícipio de Parapuã no estado de São Paulo. O ensaio foi instalado na área experimental da Casul - Cooperativa Agropecuária de Parapuã-SP, no dia 20 de setembro de 2019. O delineamento empregado foi em blocos casualizados, contendo quatro repetições. Os tratamentos constaram de oito linhagens (17-1253 OL, 18-1952 OL, 18-1968 OL, 18-2056 OL, 18-2101 OL, 18-2133 OL, 18-2136 OL e 18-2178 OL), e duas cultivares, uma desenvolvida pela Embrapa (BRS 423 OL) e outra na Argentina pelo Criadero El Carmen (Granoleico). As parcelas foram constituídas por duas linhas de três metros de comprimento, com espaçamento de $0,90 \mathrm{~m}\left(5,4 \mathrm{~m}^{2}\right.$ por parcela). Foram utilizadas práticas de manejo cultural seguindo as recomendações para as lavouras comerciais. Avaliou-se a severidade de mancha preta, massa de 100 grãos e produtividade de vagens. As menores notas de severidade de mancha preta foram obtidas nos genótipos 182056 OL, 18-1968 OL, 18-2133 OL, BRS 423 OL e 18-1952 OL. As linhagens apresentaram diferenças quanto à massa de 100 grãos, podendo atender a diferentes requisitos de granulometria do mercado e indústria. Os genótipos 17-1253 OL, 18-2101 OL e 18-2056 OL demonstraram produtividades satisfatórias para o cultivo na região da Alta Paulista.
\end{abstract}

Palavras-chave: Arachis hypogaea L.; Programa de Melhoramento; Produtividade.

\section{Agronomic performance of peanut genotypes in the Alta Paulista region}

\begin{abstract}
The peanut culture has great relevance in the agro-industrial activity in the Alta Paulista region in the state of São Paulo. This work aimed to evaluate the agronomic performance of ten peanut genotypes, in the Alta Paulista region, municipality of Parapuã in the state of São Paulo. The test was installed in the experimental area of Casul - Cooperativa Agropecuária de Parapuã-SP, on September 20, 2019. The design used was in randomized blocks, containing four replications. The treatments consisted of eight strains (17-1253 OL, 18-1952 OL, 18-1968 OL, 18$2056 \mathrm{OL}, 18-2101 \mathrm{OL}, 18-2133 \mathrm{OL}, 18-2136 \mathrm{OL}$ and 18-2178 OL), and two cultivars, one developed by Embrapa (BRS $423 \mathrm{OL}$ ) and the other in Argentina by Criadero El Carmen
\end{abstract}


(Granoleico). The plots consisted of two lines three meters long, with a spacing of $0.90 \mathrm{~m}$ (5.4 $m^{2}$ per plot). Cultural management practices were used following the recommendations for commercial crops. Severity of black spot, mass of 100 grains and pod yield were evaluated. The lowest grades of black spot severity were obtained in the genotypes 18-2056 OL, 18-1968 OL, 18-2133 OL, BRS 423 OL and 18-1952 OL. The strains showed differences regarding the mass of 100 grains, being able to meet different granulometry requirements of the market and industry. The genotypes 17-1253 OL, 18-2101 OL and 18-2056 OL showed satisfactory yields for cultivation in the Alta Paulista region.

Keywords: Arachis hypogaea L.; Improvement Program; Productivity.

\title{
Rendimiento agronómico de genotipos de maní en la región de Alta Paulista
}

\section{RESUMEN}

\begin{abstract}
La cultura del maní tiene una gran relevancia en la actividad agroindustrial en la región de Alta Paulista en el estado de São Paulo. El objetivo de este trabajo fue evaluar el desempeño agronómico de diez genotipos de maní, en la región de Alta Paulista, municipio de Parapuã en el estado de São Paulo. La prueba se instaló en el área experimental de Casul - Cooperativa Agropecuária de Parapuã-SP, el 20 de septiembre de 2019. El diseño utilizado fue en bloques aleatorios, que contenían cuatro repeticiones. Los tratamientos consistieron en ocho cepas (17-1253 OL, 18-1952 OL, 18-1968 OL, 18-2056 OL, 18-2101 OL, 18-2133 OL, 18-2136 OL y 18$2178 \mathrm{OL}$ ), y dos cultivares, uno desarrollado por Embrapa (BRS $423 \mathrm{OL}$ ) y el otro en Argentina por Criadero El Carmen (Granoleico). Las parcelas consistieron en dos líneas de tres metros de largo, con una separación de $0.90 \mathrm{~m}$ (5.4 $\mathrm{m}^{2}$ por parcela). Se utilizaron prácticas de manejo cultural siguiendo las recomendaciones para cultivos comerciales. Se evaluaron la gravedad de la mancha negra, la masa de 100 granos y el rendimiento de la vaina. Los grados más bajos de severidad de la mancha negra se obtuvieron para los genotipos 18-2056 OL, 18-1968 OL, 182133 OL, BRS 423 OL y 18-1952 OL. Las cepas mostraron diferencias con respecto a la masa de 100 granos, pudiendo cumplir con los diferentes requisitos de granulometría del mercado y la industria. Los genotipos 17-1253 OL, 18-2101 OL y 18-2056 OL mostraron rendimientos satisfactorios para el cultivo en la región de Alta Paulista.
\end{abstract}

Palabras clave: Arachis hypogaea L.; Programa de Mejoramiento; Productividad.

\section{Introdução}

A cultura do amendoim (Arachis hypogaea L.) na região da Alta Paulista tem ganhado importância no setor agroindustrial (BAZANELLA et al., 2019). A região se destaca pela área cultivada, altas produtividades e tecnologia aplicada aos campos de produção. Nos últimos anos, foram realizados diversos investimentos no parque agroindustrial, como secagem artificial, armazenamento, beneficiamento, classificação e blancheamento de amendoim. Também foram implementadas normas para atender certificações internacionais e aspectos sanitários, incluindo o monitoramento da presença de aflatoxinas e de resíduos de agroquímicos, para garantir a 
qualidade e atendimento das exigências do mercado de exportação (SAMPAIO, 2019).

A manutenção e a ampliação do espaço brasileiro nesses mercados têm relação direta com pesquisa e desenvolvimento de novas cultivares de amendoim (VIZEU, 2019). O desenvolvimento de novas cultivares é um fator determinante para o sucesso da cultura do amendoim, visando encontrar genótipos mais competitivos e adaptados às condições edafoclimáticas regionais, incluindo produtividade e padrão de vagens, grãos tipo runner e característica "alto oleico" (RIBEIRO et al., 2017; 2018).

A Cooperativa Casul em 2018 tornou-se produtora de sementes certificadas de amendoim, cadastrada no Registro Nacional de Sementes e Mudas (RENASEM) do Ministério da Agricultura, Pecuária e Abastecimento (MAPA), trazendo para seus cooperados sementes de elevada pureza genética. Em junho de 2019, estabeleceu-se um convênio com a Empresa Brasileira de Pesquisa Agropecuária (EMBRAPA), parceria essa de grande valia para o setor, conduzindo experimentos que visam avaliar novas cultivares e linhagens (COOPERATIVA CASUL, 2020).

Este trabalho teve como objetivo avaliar o desempenho agronômico de dez genótipos de amendoim, na região da Alta Paulista, munícipio de Parapuã no estado de São Paulo.

\section{Material e métodos}

O experimento foi realizado na área experimental da Cooperativa Casul, localizada na Região Oeste do estado, no município de Parapuã-SP, ano agrícola 2019/20, sendo implantado no dia 20 de setembro de 2019.

O delineamento empregado foi em blocos casualizados com quatro repetições. Os tratamentos constaram de oito linhagens (17-1253 OL, 181952 OL, 18-1968 OL, 18-2056 OL, 18-2101 OL, 18-2133 OL, 18-2136 OL e 18-2178 OL), e duas cultivares, uma desenvolvida pela Embrapa (BRS 423 $\mathrm{OL}$ ) e outra na Argentina pelo Criadero El Carmen (Granoleico). As parcelas foram constituídas por duas linhas de três metros de comprimento, com espaçamento de 0,90 $\mathrm{m}$ e área útil para avaliação de $5,4 \mathrm{~m}^{2}$ por parcela. 
A correção do solo, adubação e gessagem foi realizada conforme a análise de solo e o manejo fitossanitário (fungicidas e inseticidas) de acordo com as recomendações adequadas para a cultura. $\mathrm{O}$ controle de plantas invasoras foi realizado por meio de capina manual, sendo assim não foi utilizado herbicida. O estande médio de plantas foi de 17 plantas $/ \mathrm{m}^{-1}$.

Após 120 dias do plantio (DAP), foi realizada avaliação de severidade de mancha preta (Cercosporidium personatum), usando uma escala diagramática da incidência com notas de 1 a 9 , em que 1 indicava a planta sem doença e 9 desfolha intensa (SUBRAHMANYAM et al., 1982). A inversão das parcelas ocorreu aos 145 DAP e após a secagem, foram feitas as avaliações de massa de 100 grãos $(\mathrm{g})$ e produtividade de vagens $\left(\mathrm{kg} / \mathrm{ha}^{-1}\right.$ e sacas/alqueire ${ }^{-1}$ ).

Os dados foram submetidos à análise de variância pelo Teste $\mathrm{F}$ e as médias dos tratamentos foram comparados pelo teste de Scott-Knott, no nível de $p \geq 0,1$ de probabilidade, por meio do programa computacional SISVAR 5.6 (FERREIRA, 2019).

\section{Resultados e discussão}

Houve diferença significativa para todas variáveis. Na Tabela 1, podemos observar que os genótipos de amendoim estão ordenados de acordo com os valores fenotípicos de cada variável. Sobre a severidade de mancha preta (notas), observou-se que apesar do controle químico das doenças foliares, houve desfolha intensa dos terços inferior e médio devido à ocorrência de cercosporioses em todos os genótipos. Provavelmente a desfolha intensa tenha ocorrido em função da ocorrência de períodos longos de elevada precipitação na época da colheita nesta safra.

Todos os genótipos apresentaram severidade com nota acima de 7,0. Os genótipos 18-2056 OL (7,2), 18-1968 OL $(7,3), 18-2133$ OL $(7,3)$, BRS 423 OL $(7,5)$ e 18-1952 OL $(7,6)$ apresentaram maior severidade. Os resultados corroboram com Bazanella et al. (2019) que obtiveram efeitos semelhantes de severidade com o genótipo BRS 423 OL $(7,6)$, e esteve entre as menores notas do ensaio, utilizando o mesmo arranjo de semeadura, no munícipio de Tupã-SP. A maior severidade foi encontrada no 
genótipo 18-2101 OL $(8,2)$, resultado esse que foi semelhante ao observado por Martins et al. (2019), que também obtiveram alta severidade ao avaliar o genótipo 18-2101 OL, com a nota 8,4, em Santo Antônio de Goiás-GO.

Tabela 1. Severidade de mancha preta (notas), massa de 100 grãos (g) e produtividade de vagens $\left(\mathrm{kg} / \mathrm{ha}^{-1}\right.$ e sacas/alqueire $\left.{ }^{-1}\right)$ em função de diferentes genótipos de amendoim na região da Alta Paulista. Parapuã-SP, 2019/20.

\begin{tabular}{|c|c|c|c|c|}
\hline \multirow{2}{*}{$\begin{array}{l}\text { Linhagens/ } \\
\text { Cultivares }\end{array}$} & \multirow{2}{*}{$\begin{array}{l}\text { Severidade } \\
\text { (notas) }\end{array}$} & \multirow{2}{*}{$\begin{array}{l}\text { Massa de } \\
100 \text { grãos (g) }\end{array}$} & \multicolumn{2}{|c|}{ Produtividade de vagens } \\
\hline & & & $\left(\mathrm{kg} / \mathrm{ha}^{-1}\right)$ & (sacas/alqueire $\left.^{-1}\right)$ \\
\hline $17-1253 \mathrm{OL}$ & $8,1 \mathrm{~b}$ & 84,3 a & $7.655,5 \mathrm{a}$ & 741,0 \\
\hline $18-2101 \mathrm{OL}$ & $8,2 \mathrm{~b}$ & $78,7 \mathrm{c}$ & $7.101,8 \mathrm{a}$ & 687,5 \\
\hline $18-2056 \mathrm{OL}$ & $7,2 \mathrm{a}$ & $77,9 d$ & $7.017,0 \mathrm{a}$ & 679,2 \\
\hline $18-2133 \mathrm{OL}$ & $7,3 \mathrm{a}$ & $76,8 \mathrm{e}$ & $6.546,0 \mathrm{~b}$ & 633,7 \\
\hline 18-1952 OL & $7,6 \mathrm{a}$ & $79,5 b$ & $6.494,9 \mathrm{~b}$ & 628,7 \\
\hline $18-1968 \mathrm{OL}$ & $7,3 \mathrm{a}$ & $74,8 \mathrm{~g}$ & $6.362,4 \mathrm{~b}$ & 615,9 \\
\hline Granoleico & $7,9 \mathrm{~b}$ & $78,8 \mathrm{c}$ & $6.330,8 \mathrm{~b}$ & 612,8 \\
\hline BRS $423 \mathrm{OL}$ & $7,5 \mathrm{a}$ & $78,0 \mathrm{~d}$ & $6.261,2 \mathrm{~b}$ & 606,1 \\
\hline $18-2178 \mathrm{OL}$ & $7,8 \mathrm{~b}$ & $75,0 \mathrm{~g}$ & $6.190,4 \mathrm{~b}$ & 599,2 \\
\hline $18-2136 \mathrm{OL}$ & $7,8 \mathrm{~b}$ & $75,5 f$ & $6.185,1 \mathrm{~b}$ & 598,7 \\
\hline Média & 7,7 & 77,9 & $6.614,5$ & 640,3 \\
\hline C.V. (\%) & 6,3 & 0,6 & 11,7 & - \\
\hline $\mathrm{Pr}>\mathrm{Fc}$ & $0,0552^{\star}$ & $>0,0001^{*}$ & $0,1652^{*}$ & - \\
\hline
\end{tabular}

A maior massa de 100 grãos (g) foi obtida com o genótipo 17-1253 OL (84,3 g) e a menor com o 18-1968 OL (74,8 g), obtendo diferença de 9,5 g. Ribeiro et al. (2018) também observaram diversidade no tamanho dos grãos, obtendo uma diferença de $15,1 \mathrm{~g}$ em relação aos genótipos de maior e menor massa (JS 21406 e 16-822 OL, respectivamente), em Tupã-SP. O genótipo que apresentou maior massa de 100 grãos (17-1253 OL) também foi o que obteve a maior produtividade.

Em relação a produtividade de vagens $\left(\mathrm{kg} / \mathrm{ha}^{-1}\right)$, observa-se que as maiores produtividades foram obtidas pelas linhagens $17-1253 \mathrm{OL}(7.655,5$ $\left.\mathrm{kg} / \mathrm{ha}^{-1}\right), 18-2101 \mathrm{OL}\left(7.101,8 \mathrm{~kg} / \mathrm{ha}^{-1}\right)$ e $18-2056 \mathrm{OL}\left(7.017,0 \mathrm{~kg} / \mathrm{ha}^{-1}\right)$, com produtividades superiores a $7.000 \mathrm{~kg} / \mathrm{ha}^{-1}$. A produtividade média do experimento foi de $6.614,5 \mathrm{~kg} / \mathrm{ha}^{-1}$. Estes resultados demonstram que as 
cultivares Granoleico e BRS $423 \mathrm{OL}$ apresentaram produtividades inferiores as novas linhagens (17-1253 OL, 18-2101 OL e 18-2056 OL).

Estes resultados ressaltam a importância do programa de melhoramento genético e a pesquisa científica em campo, apresentando ganhos gradativos em produção pelo processo de seleção de novas cultivares. Outros estudos semelhantes a estes deverão ser realizados, visando dar continuidade a seleção de genótipos para região. A linhagem 18-2056 OL obteve uma das maiores produtividades, sendo a que apresentou menor nota de severidade de mancha preta, demonstrando seu potencial produtivo aliado a resistência a $C$. personatum para cultivo na região da Alta Paulista

\section{Conclusões}

As menores notas de severidade de mancha preta foram obtidas nos genótipos 18-2056 OL, 18-1968 OL, 18-2133 OL, BRS 423 OL e 18-1952 OL. As linhagens apresentaram diferenças quanto à massa de 100 grãos, podendo atender a diferentes requisitos de granulometria do mercado e indústria. Os genótipos 17-1253 OL, 18-2101 OL e 18-2056 OL demonstraram produtividades satisfatórias para o cultivo na região da Alta Paulista.

\section{Agradecimentos}

Os autores agradecem à CASUL - Cooperativa Agropecuária de Parapuã-SP, pelo suporte financeiro e espaço físico para condução do presente estudo. Este trabalho também está vinculado ao Programa de Melhoramento do Amendoim da Embrapa (SEG 20.18.01.021.00). 


\section{Referências}

BAZANELLA, M. R.; HEUERT, J.; MARTINS, K. B. B.; SUASSUNA, T. M. F.; DEUS, T. J. Desempenho de genótipos de amendoim na Alta Paulista com avaliação de perdas na colheita. In: Anais do Encontro Sobre a Cultura do Amendoim, 16., 2019, Jaboticabal. Anais eletrônicos... Campinas: GALOÁ, 2019. Disponível em: <https://proceedings.science/encontro-amendoim2019/papers/desempenho-de-genotipos-de-amendoim-na-alta-paulista-comavaliacao-de-perdas-na-colheita> Acesso em: 19 abr. 2020.

COOPERATIVA CASUL. Sementes certificadas. 2020. Disponível em: <https://www.casul.com.br/amendoim/> Acesso em: 17 abr. 2020.

FERREIRA, D. F. SISVAR: a computer analysis system to fixed effects split plot type designs. Revista Brasileira de Biometria, [S.L.], v. 37, n. 4, p. 529, 20 dez. 2019. http://dx.doi.org/10.28951/rbb.v37i4.450.

MARTINS, K. B. B.; RODRIGUES, L. L.; HEUERT, J.; XAVIER, M. F. N.; SUASSUNA, T. M. F.; BETIOL, R. A. B. Desempenho agronômico de novas linhagens de amendoim no Cerrado. In: Anais do Encontro Sobre a Cultura do Amendoim, 16., 2019, Jaboticabal. Anais eletrônicos... Campinas: GALOÁ, 2019. Disponível em: <https://proceedings.science/encontroamendoim-2019/papers/desempenho-agronomico-de-novas-linhagens-deamendoim-no-cerrado> Acesso em: 19 abr. 2020.

RIBEIRO, R. P.; HEUERT, J; SUASSUNA, N. D.; SUASSUNA, T. M. F. Desempenho de Linhagens de amendoim sob alta severidade de doenças. In: Anais do Encontro Sobre a Cultura do Amendoim, 14., 2017, Jaboticabal. Anais eletrônicos... Campinas: GALOÁ, 2017. Disponível em: $<$ https://proceedings.science/encontro-amendoim-2017/papers/desempenhode-linhagens-de-amendoim-sob-alta-severidade-de-doencas-

foliares?lang=pt-br> Acesso em: 18 abr. 2020.

RIBEIRO, R. P.; SUASSUNA, T. M. F.; HEUERT, J.; SOAVE, J. H.; SANTOS, L. C. C. Desempenho de genótipos de amendoim na Alta Paulista. In: Anais do Encontro Sobre a Cultura do Amendoim, 15., 2018, Jaboticabal. Anais eletrônicos... Campinas: GALOÁ, 2018. Disponível em: $<$ https://proceedings.science/encontro-amendoim-2018/papers/desempenhode-genotipos-de-amendoim-na-alta-paulista> Acesso em: 18 abr. 2020.

SAMPAIO, R. M. Amendoim: Evolução da produção e comercialização na última década. In: SILVA, R. P.; SANTOS, A. F.; CARREGA, W. C. Avanços na produção de amendoim. 1. ed. Jaboticabal-SP: Funep, 2019, p. 17-23.

SUBRAHMANYAM, P.; MCDONALD, D.; GIBBONS, R. W.; NIGAM, S. N.; NEVILL, D. J. Resistance to rust and late leaf spot diseases in some genotypes of Arachis hypogaea. Peanut Science, v. 9, n. 1, p. 6-10, 1982. https://doi.org/10.3146/i0095-3679-9-1-2.

VIZEU, L. A. Revista Canavieiros. Panorama da cadeira produtiva do amendoim. n. 159, p. 12-13, set. 2019. Disponível em: 
$<$ https://www.revistacanavieiros.com.br/uploads/pagina/tag/2019/11/e8|GHvh bxTeXEgc9/159-set-baixa.pdf>. Acesso em: 18 abr. 2020. 